\title{
BMJ Open Effect of vitamin D supplementation on pain and physical function in patients with knee osteoarthritis (OA): an OA Trial Bank protocol for a systematic review and individual patient data (IPD) meta-analysis
}

Xingzhong Jin (1) , ${ }^{1,2,3}$ Benny Antony, ${ }^{4}$ Xia Wang, ${ }^{3}$ Monica SM Persson (D) , Timothy McAlindon, ${ }^{6}$ Nigel K Arden, ${ }^{7,8}$ Sudeepti Srivastava, ${ }^{9}$ Rajeshwar Srivastava, ${ }^{9}$ Marienke Van Middelkoop, ${ }^{10}$ Sita MA Bierma-Zeinstra, ${ }^{10}$ Weiya Zhang, ${ }^{11}$ Flavia Cicuttini, ${ }^{12}$ Changhai Ding ${ }^{1,4,12}$

To cite: Jin $X$, Antony $B$, Wang $X$, et al. Effect of vitamin $D$ supplementation on pain and physical function in patients with knee osteoarthritis $(\mathrm{OA})$ an OA Trial Bank protocol for a systematic review and individual patient data (IPD) meta-analysis. BMJ Open 2020;10:e035302. doi:10.1136/ bmjopen-2019-035302

- Prepublication history and additional material for this paper are available online. To view these files, please visit the journal online (http://dx.doi. org/10.1136/bmjopen-2019035302).

Received 27 October 2019 Revised 06 March 2020 Accepted 26 March 2020

Check for updates

(C) Author(s) (or their employer(s)) 2020. Re-use permitted under CC BY-NC. No commercial re-use. See rights and permissions. Published by BMJ.

For numbered affiliations see end of article.

Correspondence to Dr Xingzhong Jin; xingzhong.jin@unsw.edu.au and Dr Changhai Ding;

Changhai.Ding@utas.edu.au

\section{ABSTRACT}

Introduction Observational data suggest that vitamin D deficiency is associated with the onset and progression of knee osteoarthritis (OA). However, randomised controlled trials (RCTs) to date investigating the efficacy of vitamin D supplementation in knee OA have reported conflicting results. Further research is needed to clarify the effects of vitamin D on patient-reported outcomes and determine whether there are patient subgroups who may benefit from the supplementation. The aim of this individual patient data (IPD) meta-analysis is to identify patient-level predictors of treatment response to vitamin D supplementation on pain and physical function.

Methods and analysis A systematic literature search will be conducted for RCTs of vitamin D supplementation on knee OA. Authors of original RCTs will be contacted to obtain the IPD. The primary outcomes will include longterm ( $\geq 12$ months) pain and physical function. Secondary outcomes will include medium-term $(\geq 6$ months and $<12$ months) and short-term ( $<6$ months) pain and physical function, as well as patient global assessment, quality of life and adverse events. Potential treatment effect modifiers to be examined in the subgroup analyses include age, gender, body mass index, baseline knee pain severity and physical function, baseline vitamin D level, radiographic stage, presence of bone marrow lesions on MRI, presence of clinical signs of local inflammation and concomitant depressive symptoms. Both one-step and two-step modelling methods will be used to determine the possible modifiable effect of each subgroup of interest. Ethics and dissemination Research ethical or governance approval is exempt for this study as no new data are being collected. This study will be the first IPD meta-analysis to clarify the effect of vitamin D supplementation on clinical symptoms in different subgroups of patients with knee $0 \mathrm{~A}$. The findings will be disseminated through peer-review publications and conference presentations.

PROSPERO registration number CRD42018107740.
Strengths and limitations of this study

This study protocol is endorsed by the osteoarthritis (OA) Trial Bank, an international collaboration that initiates meta-analyses on predefined subgroups of OA patients.

- This study is the first individual patient data (IPD) meta-analysis to identify $O A$ patient subgroups that may benefit from vitamin $D$ supplementation.

- IPD meta-analysis offers greater statistical power and precision than subgroup analysis in a single trial and conventional meta-analysis using aggregate data.

- Analysis may be limited to the variables that have been collected across included randomised controlled trials.

\section{INTRODUCTION}

Osteoarthritis (OA) is the most common joint disorder worldwide. OA is ranked as the 13th highest contributor of 310 diseases to global disability in 2015. ${ }^{1}$ In Australia, OA affects over 2.1 million people (9\% of the population), costing the healthcare system over $\$ 2.1$ billion in $2015 .^{2}$ This cost is forecasted to increase by $39 \%$ in $2030 .^{3}$

The majority $(83 \%)$ of the disease burden of $\mathrm{OA}$ is attributable to knee $\mathrm{OA},{ }^{4}$ which is characterised by pain, gradual loss of articular cartilage and structural changes such as subchondral remodelling and effusionsynovitis. ${ }^{5}$ Currently, there are no effective disease-modifying treatments to reverse the progression of knee OA once the disease is established. A majority of patients with knee OA eventually progress to advanced stage 
and undergo total knee replacement. ${ }^{6}$ There is clearly an urgent need for innovative and cost-effective approaches to slow the progression of knee OA.

Emerging observational data suggest that vitamin D insufficiency is associated with the onset and progression of knee OA. ${ }^{7}$ The association between low serum vitamin D levels and knee OA symptoms may be explained by a direct effect of vitamin D on chondrocytes in osteoarthritic cartilage, ${ }^{8}$ as well as indirect effects on subchondral bone, synovium and periarticular muscle. ${ }^{9}$ For example, vitamin $\mathrm{D}$ deficiency could impair the ability of bone to respond optimally to pathological bone marrow lesions (BMLs) and altered bone mineral density, therefore predisposing the knees to disease progression. ${ }^{10}$ Vitamin D also may reduce synovitis in affected knees by regulating cytokine levels in the joint, leading to a reduction in the amount of effusion-synovitis in MRI. ${ }^{11}$ In addition, depressive symptoms is interrelated to joint pain in $\mathrm{OA},{ }^{12}$ and maintaining sufficient serum vitamin D may improve depression symptoms in patients with knee OA. $^{13}$

However, evidence from randomised controlled trials (RCTs) have been conflicting. ${ }^{14-17}$ A pilot RCT in India found a small but statistically significant clinical benefit for 12-month vitamin $\mathrm{D}$ treatment on pain and function in patients with knee OA, compared with placebo. ${ }^{16} \mathrm{In}$ contrast, a subsequent RCT from the USA showed that vitamin D supplementation did not reduce knee pain or cartilage volume loss over 24 months.${ }^{15}$ Subsequently, data from RCTs in Australia ${ }^{17}$ and $\mathrm{UK}^{14}$ did not find significant clinical benefits of vitamin D supplementation for knee OA. However, there was a non-significant trend for symptom reduction (eg, knee pain and physical function) in these RCTs. Two systematic reviews using aggregate data from these RCTs concluded that, although current evidence does not support vitamin D supplementation for reducing structural disease progression, further research is needed to clarify the effects on patient-reported outcomes. ${ }^{18}{ }^{19}$ In addition, to the best of our knowledge, no previous systematic reviews of previous systematic reviews exist.

The null results of these RCTs could be due to a low prevalence of vitamin D deficiency in the study subjects or low statistical power secondary to small sample sizes. Another possible reason is that vitamin D may have an effect only in some OA phenotypes, such as those with BMLs (predominant bone abnormality), ${ }^{20}$ effusionsynovitis (inflammatory) ${ }^{21}$ or depressive symptoms (psychological distress). ${ }^{13}$ Post hoc analyses within these RCTs ${ }^{15}{ }^{21}$ were frequently underpowered, and hence unreliable to determine the effect of vitamin D treatment on subgroups of knee OA patients. A metaanalysis using individual patient data (IPD) can increase the power of subgroup analysis by combining individual data from included trials ${ }^{22}$ and therefore can quantify potential effect modifier of vitamin $D$ treatment in subgroups.

\section{METHODS AND ANALYSIS}

We will conduct a systematic review and IPD meta-analysis of RCTs studying the effects of vitamin D supplementation in patients with symptomatic knee OA. The primary aim is to identify patient-level predictors of treatment response to vitamin $\mathrm{D}$ supplementation, including the status of vitamin D deficiency, MRI-detected bone marrow abnormalities and effusion-synovitis, and clinical signs of local inflammation. The protocol of this review is registered on the PROSPERO database (CRD42018107740).

\section{Study eligibility}

This systematic review will include studies that meet the following inclusion criteria:

\section{Type of studies}

RCTs that have been published in journals and reported the efficacy of vitamin D in participants with knee OA will be included. Cross-over design will also be eligible and only the first phase data will be included in the analysis. Both individually randomised trials and cluster randomised trials will be eligible. Both open-labelled and blinded studies will be eligible. There will be no language or geographical restrictions applied to study selection.

\section{Participants}

Men and women who have a diagnosis of knee OA, either according to the American College of Rheumatology criteria, or on the basis of detailed clinical and/or radiographic information, will be included. Studies with a subgroup of knee OA patients will also be included, provided that IPD can be collected separately for the OA subgroup. Although most patients with knee OA defined by the American College of Rheumatology are usually over 50 years of age, the disease can occur as early as 20 years old, therefore studies with adults at 18 years of age and older will be included.

\section{Interventions}

Only studies investigating oral supplementation of vitamin $\mathrm{D}$ will be included. Vitamin $\mathrm{D}$ treatments administered subcutaneously, intraperitoneally or intravenously will not be included. Both ergocalciferol $\left(\right.$ vitamin $\mathrm{D}_{2}$ ) and cholecalciferol (vitamin $\mathrm{D}_{3}$ ) will be eligible, irrespective of preparations (tablet or capsule), dosage, regimen and length of treatment.

\section{Comparators}

Oral vitamin D supplementation will be compared with control treatment including placebo or usual conservative care (pain medication and/or exercise therapy if they are used in both treatment and control groups).

\section{Outcomes}

The minimum criterion for inclusion is reporting pain or physical function as either primary or secondary outcomes. There will be no restrictions on the duration of follow-up. Data on other outcomes (eg, patient's global assessment, quality of life and adverse events) will be 
analysed when feasible but will not be required for study selection.

\section{Baseline assessments}

As a minimum, included studies should have measured knee pain, physical function, serum levels of vitamin $\mathrm{D}$ at baseline, and included basic patient characteristics such as age, gender and body mass index (BMI).

\section{Literature search}

A systematic literature search for articles published from 1 January 1990 until 31 December 2019 will be performed by a trained review author $(\mathrm{XJ})$ in the following electronic databases:

- Cochrane Central Register of Controlled Trials (CENTRAL).

- MEDLINE (Ovid).

- EMBASE.

A previous systematic review ${ }^{7}$ has showed that no major RCTs were conducted for vitamin D and OA by the year 1990, therefore we chose 1 January 1990 as the start date of the literature search. The search strategies used for each database are listed in online supplementary appendix 1 . All retrieved articles will be exported to the reference manager EndNote, in which duplicates will be removed electronically and manually. The remaining records will be exported to an online systematic review management tool Covidence (Veritas Health Innovation, Melbourne, Australia) and the articles will be assessed for eligibility for inclusion. In addition to the electronic search, we will check the reference lists of included trials and previous systematic reviews to identify any trials that are not retrieved from the electronic search. Review authors and collaborating authors will be asked if they are aware of further relevant studies not yet included. We will also search the WHO International Clinical Trial Registration Platform Search Portal (www.who.int/trialsearch) to identify any relevant trials that are completed but did not published the results.

\section{Study selection}

Two review authors (XJ, BA) will independently conduct study screening by assessing the article titles and abstracts. Full texts of the articles will be further assessed if the information from the abstract suggests that a study is eligible for inclusion. When information contained in the full text is not sufficient to make a judgement on its eligibility, we will make efforts to contact the corresponding authors to obtain further details. If a corresponding author is not contactable after two email attempts and one phone call, the study will be deemed ineligible. Any disagreement regarding the inclusion of a study will be discussed between the two review authors (XJ, BA). If no consensus can be reached, a third review author will be consulted (XW) to make a final decision.

\section{Extraction of aggregate data}

Study data extraction will be performed independently by two review authors (BA, XW). Discrepancies will be resolved by a third reviewer $(\mathrm{XJ})$. We will extract the following study data from the included studies:

- General information: article title, bibliographic details, published language and funding source.

- Participants: inclusion criteria, exclusion criteria, number of participants in total and in each study arm, study settings and baseline participant characteristics (eg, age, gender, BMI).

- Intervention: type of vitamin D preparation, dose, regimen, treatment duration, type of control and co-interventions (if any).

- Outcomes: primary and secondary outcomes at the end of treatment and/or the end of follow-up. Number of withdrawals and loss to follow-up. Adverse events recorded.

- Data analysis: statistical models used for data analysis, confounding factors adjusted in the models and methods used for addressing missing values.

\section{Risk of bias assessment}

The methodological quality of the included studies will be assessed using a modified version of the Cochrane's risk of bias tool. ${ }^{23}$ The modified version includes all the seven domains of the original tool, but further separates performance bias assessment into 'blinding of participants' and 'blinding of study personnel', as well as prespecifies other sources of bias as 'balance in baseline covariates', 'treatment compliance' and 'timely outcome assessment' (online supplementary appendix 2). Each domain will be scored as 'low risk', 'high risk' or 'unclear' according to the criteria described in the online supplementay appendix 3. The overall rating of risk of bias is based on the number of domains with a 'high-risk' score. The overall rating is intended to inform readers of the risk of bias across individual studies and will not be used to weight the studies in the meta-analysis. Two review authors (XJ, XW) will independently evaluate the quality of an individual study. Any disagreement will be settled by further discussion until a consensus is reached between the two review authors.

\section{IPD collection and transfer}

The primary or corresponding authors of included trials will be invited to collaborate on the project and contribute their raw data. When we cannot reach a corresponding author after sending two emails and making two telephone calls, we will contact the co-authors listed in the article. If none of the co-authors can be contacted, we will approach the institutes, in which the trial has been conducted. All data custodians will be asked to sign a data delivery agreement, which includes items regarding IPD delivery, obligations, ownership of data, terms and conditions of the use of the data, authorship and publications. If needed, the project coordinator $(\mathrm{XJ})$ will visit the institutes of the data deliverers to retrieve the data and to sign the data delivery agreement on behalf of the OA Trial Bank (the detailed procedures of data delivery and an example of the data delivery agreement can be 
found on the OA Trial Bank website https://www.oatrialbank.com/procedures/). De-identified datasets will be accepted in any electronic format (eg, SPSS, Stata, SAS, Excel) or in paper form, provided that variables and categories are adequately labelled within the dataset or within a separate codebook. The IPD files received by the coordinator will be kept in their original version and saved on a secured password-protected server at Erasmus MC Medical University in Rotterdam. The datasets will not be used for any other research apart from that described in the license agreement.

To ensure the quality of the data, the coordinator will independently check for data consistency by comparing the summary statistics derived from the IPD received against the summary results reported in the published articles. In the case of differences, the project coordinator will communicate with the data deliverer via email or teleconference to resolve the discrepancy.

\section{Variables of interest}

The following IPD variables will be obtained (where available):

\section{Primary outcome variables}

The primary outcomes for this meta-analysis will be pain and physical function at long-term follow-up (12 months or more). This definition of 'long-term effect' for knee $\mathrm{OA}$ treatment was used in previous systematic reviews of knee OA research. ${ }^{24} 25$

- Knee pain will be evaluated using visual analogue scale if available, otherwise the pain subscales of the Western Ontario and McMaster Universities Osteoarthritis Index (WOMAC) ${ }^{26}$ or the Knee injury and Osteoarthritis Outcome Score (KOOS) ${ }^{27}$ will be used. The pain data will be converted into a $0-100$ common scale as recommended by the WOMAC manual. ${ }^{28}$

- Physical function will be evaluated using a validated instrument specific to knee OA, such as the physical function subscales of the WOMAC or the KOOS subscales. The scores will be standardised into a $0-100$ scale.

\section{Secondary outcome variables}

Secondary outcomes will include:

- Medium-term (more than 6 months but less than 12 months) pain and physical function.

- Short-term (less than 6 months) pain and physical function.

- Patient global assessment at the end of study follow-up, as recommended by the Outcome Measures in Arthritis Clinical Trials - Osteoarthritis Research Society International (OMERACT-OARSI) Initiative. ${ }^{29}$

- Quality of life evaluated using a validated instrument, such as EuroQol-5D (EQ-5D), ${ }^{30}$ osteoarthritis Knee and Hip Quality of Life, ${ }^{31}$ or original instrument used in the included studies.
- Adverse events if reported, including all major and minor events such as hypocalcaemia, fractures and depression.

\section{Potential treatment effect moderators}

If data are available, we will analyse potential treatment effect modification for the following variables measured at baseline:

- Radiographic stage of knee OA (mild/moderate or severe). Radiographic knee OA should be staged at baseline using either the Kellgren-Lawrence $(\mathrm{KL})^{32}$ or Osteoarthritis Research Society International (OARSI) joint space narrowing (JSN) grading system. ${ }^{33}$ The results from the two grading systems have been shown to be highly correlated. ${ }^{34}$ Mild to moderate disease will be defined as a KL Score $\leq 3$ or an OARSI JSN Score $\leq 2$, and severe disease will be a KL score of 4 or an OARSI score of 3.

- Vitamin D level (deficiency, insufficiency or sufficiency). Vitamin $\mathrm{D}$ deficiency is defined as serum levels of $25(\mathrm{OH}) \mathrm{D}$ less than $30 \mathrm{nmol} / \mathrm{L}$ at baseline. Insufficiency is defined as serum $25(\mathrm{OH}) \mathrm{D}$ levels from 30 to $<50 \mathrm{nmol} / \mathrm{L}$. Serum $25(\mathrm{OH}) \mathrm{D}$ levels $\geq 50 \mathrm{nmol} / \mathrm{L}$ is considered as vitamin D sufficiency. ${ }^{35}$

- Presence of BMLs (yes or no). BMLs at the patellafemoral and femoro-tibial joints should be measured by MRI using a validated scoring system, such as Boston Leeds Osteoarthritis Knee Score ${ }^{36}$ WholeOrgan MRI Score ${ }^{37}$ or Knee Osteoarthritis Scoring System. $^{38}$

- Clinical signs of local inflammation or the presence of effusion-synovitis (yes or no). Clinical signs of local inflammation should be assessed by physical examination-tumour (swelling), dolor (pain), rubor (redness), calor (heat) and functio laesa (disturbance of function) — or by additional laboratory testing (eg, serum $\mathrm{C}$ reactive protein and erythrocyte sedimentation rate). Effusion-synovitis should be measured on either ultrasound or MRI.

- Presence of depressive symptoms or comorbid depression. Depressive symptoms are measured using a validated questionnaire, such as the Patient Health Questionnaire- $9^{39}$ and the Geriatric Depression Scale. ${ }^{40}$

\section{Data synthesis and statistical methods}

All statistical analysis will be conducted using R V.3.5.0 and RStudio (V.1.0.136, RStudio, Boston, Massachusetts, USA), with R extension package 'metafor' for the conventional meta-analysis and two-stage IPD meta-analysis, and 'Ime4' for the one-stage IPD meta-analysis.

\section{Conventional meta-analysis}

An aggregate data meta-analysis, using a random-effects model based on the 'Hartung-Knapp-Sidik-Jonkman' method, ${ }^{41}$ will be performed to estimate the treatment effect of vitamin D over control. If an included study is a cluster RCT, results will be corrected using previously 
established procedures. ${ }^{42}$ The results will be compared with the IPD meta-analysis findings as sensitivity analyses.

Heterogeneity will be assessed by inspecting the forest plots and tested by $\chi^{2}$ test. A result of $\mathrm{p}<0.10$ will be defined as evidence of significant heterogeneity across studies. $\mathrm{I}^{2}$ test will be used to estimate the extent of inconsistency across studies that is due to heterogeneity. ${ }^{43} \mathrm{~A}$ result of over $30 \%$ and $50 \%$ represents moderate and substantial heterogeneity, respectively. Sources of heterogeneity will be explored by excluding individual trials causing an $\mathrm{I}^{2}$ score below $50 \%$.

If 10 trials or more trials are available, we will use a Doi plot (normal quantile vs effect size plot) explore publication bias and 'small-study effects'. ${ }^{44}$ Asymmetry in the Doi plot will be detected and quantified by the Luis FuruyaKanamori (LFK) index. A value beyond \pm 1 will be deemed consistent with asymmetry, thus having considerable small-study effects. ${ }^{44}$

\section{IPD meta-analysis}

IPD from included trials will be recoded and formatted in a consistent way to permit re-analysis. A new variable will be created to indicate the trial in which the IPD are collected. The method used to handle missing data will depend on the mechanism causing the missingness. If no explanation is known for the reason of missing data, they will be assumed to be missing at random. We will use the R MICE package ${ }^{45}$ for multiple imputation, and the imputation model will include all available patient variables to help predict missing data for the variables of interest within each study dataset. The imputation procedure will use 20 imputed datasets. A sensitivity analyses will be performed restricting to participants without missing data (complete case analysis).

Both the treatment effect of vitamin D supplementation and the effect of potential moderators will be studied in the IPD meta-analyses. The treatment effect of vitamin D will be measured using the mean difference in knee pain and physical function between treatment and control based on the intention-to-treat principle. The interaction between the treatment and a potential moderator will be used to identify the effect of the moderator. Interaction effects with $\mathrm{p}<0.05$ will be considered statistically significant and 95\% CIs of the effects will be provided. The IPD meta-analyses will be undertaken using both the onestage and two-stage approaches. We will use the one-stage approach as the primary analysis to avoid assumptions of within-study normality and known within-study variance. ${ }^{46}$ We will and compare the results to the two-stage approach to assess consistency in a sensitivity analysis.

\section{One-stage modelling}

The one-stage mixed-effects IPD meta-analysis approach will take into account both study level and subject-level covariates. Subject-level covariates will be centred to the mean of the covariate in each trial to avoid ecological bias. Three multilevel regression models will be built, the first to examine the summary treatment effect (difference between vitamin $\mathrm{D}$ and control), the second to evaluate each of the mentioned moderators on treatment effect and the third to assess the true effect of one moderator independent of other moderators.

The first model will include outcome measure (eg, pain score at follow-up) as a dependent variable, treatment (vitamin D or control), baseline subject-level covariate (eg, pain score) and confounders (age, gender and BMI) as fixed-effect independent variables, adjusted for study identifier (random intercept). The partial regression coefficient of the treatment will be used to compare to the conventional meta-analysis.

The second model will further add the moderator of interest (eg, radiographic stage of the disease) and interaction term between the treatment and study-centred values of the moderator in the fixed effect of the first model. The regression coefficient of the interaction term will be used to quantify the impact of the moderator on treatment effect.

If there are two or more moderators that have a statistically significant interaction with the treatment effect, these moderators will be incorporated in the third model. Multicollinearity between moderators will be tested before building the third model. A correlation coefficient $r>0.80$ will indicate that multiple collinearity exists between the two moderators, of which the one that has less measurement error will be included in the model.

\section{Two-stage modelling}

In the first stage, treatment effect and variance are derived from separate analysis in each study. An analysis of covariance model adjusted for baseline covariates (age, gender, BMI, as well as knee pain or function), will be used to obtain the treatment effect and variance within each study separately. The effect of a moderator and its variance within each study will be obtained by adding the interaction term between treatment effect and the moderator into the model. In the second stage, the treatment effect and its variances obtained from the first stage will be pooled across studies using a random-effects model. ${ }^{41}$ The result of this model is a summary estimate of the treatment effect of vitamin $\mathrm{D}$ versus control. The effect of a moderator will be calculated by pooling the regression coefficient and variance of the interaction term between the treatment and the moderator using a similar model. If two or more interaction terms are statistically significant, these moderators will be incorporated in a further model to evaluate the independent effect of these moderators.

\section{Patient and public involvement}

We do not directly include patient and public involvement in this study, but the design of included RCTs may involve patients.

\section{DISCUSSION}

Vitamin D supplementation may improve pain and function in patients with knee OA; however, the evidence 
from observational studies and RCTs are controversial. ${ }^{718}$ In a few existing RCTs in patients with symptomatic knee OA, ${ }^{141617}$ small to modest improvement in knee pain and physical function were observed in participants receiving vitamin $\mathrm{D}$ supplementation, but the treatment response varies considerably and the effects were not statistically significant when compared with placebo. The broad variation in treatment response could be a result of the fact that knee OA is a highly heterogenous disease with multiple aetiologies. ${ }^{47}$ Vitamin D may therefore be more effective in a subset of patients with specific characteristics than others. The purpose of the proposed IPD metaanalysis is to evaluate the treatment response of vitamin D supplementation on pain and physical function for knee $\mathrm{OA}$ in specific subsets of patients according to disease severity, vitamin D levels, presence of BMLs, inflammatory signs and depressive symptoms, over both short-term and long-term follow-up.

Meta-analysis is considered by some to be the grandmother of 'big data" ${ }^{48}$ and an integrated part of precision medicine. ${ }^{49}$ It is a cost-effective approach to identify potential moderators of treatment response at a patient level, which is not possible with subgroup analyses in a single trial or conventional meta-analysis with aggregate data. ${ }^{50}$ Subgroup analyses within individual trials are often underpowered to generate reliable findings. In contrast, IPD meta-analysis techniques increase the statistical power of the study by combining individual observation data from multiple trials with the same treatment. ${ }^{22}$ This offers greater precision in analysing moderators of treatment response and offers the potential to analyse a greater number of moderators. In conventional metaanalysis, meta-regression analysis may be used to examine differences in the types of patients enrolled in individual trials, but is potentially problematic. Meta-regression analysis may make incorrect inferences about individual characteristics based on aggregate baseline statistics reported in trial publications. ${ }^{51}$ This is also known as ecological fallacy, ${ }^{52}$ where the relationship between the effect estimate and average patient characteristics across trials may not be the same as the relationship within individual trials.

Identifying different phenotypes of knee OA is currently a popular subject of research in the field. Different phenotyping strategies have been proposed based on risk factors from epidemiological studies,${ }^{53}$ anatomical abnormalities on modern imaging ${ }^{54}$ or molecular abnormalities related to pathological mechanisms. ${ }^{55}$ While there is currently no standardised classification system for knee OA phenotypes, ${ }^{5}$ we believe that disease phenotypes are most meaningful when they reflect differential treatment effects. IPD meta-analysis provides an opportunity to assess the effect of treatments on subtypes or phenotypes of patients with knee OA according to predefined set of characteristics. The current proposed IPD metaanalysis attempts to differentiate subgroups by identifying subtypes of patients that respond better to vitamin D supplementation on pain and physical function.

\section{Strengths and limitations}

Several challenges may present when conducting an IPD meta-analysis. First, although IPD meta-analyses usually offer sufficient statistical power to examine moderators of treatment response, not all RCTs measure potential moderators of interest or measure them in the same way. This may limit the analysis to only exploring moderators that have been collected across studies. The current protocol attempts to minimise this risk by including moderators that are commonly reported in OA research. In addition, there are expected barriers to accessing data, such as the authors of included trials not being able to be contacted, or the authors losing access to raw data or not choosing to collaborate, may introduce selection bias into the IPD meta-analysis. However, this could be examined in sensitivity analyses by comparing the results to the conventional meta-analysis or be addressed using frameworks that combine IPD and aggregate data in a meta-analysis. ${ }^{56}$

The benefits of using IPD meta-analysis techniques greatly outweigh the challenges of using this intensive method. Previous RCTs and systematic reviews have not had sufficient power to thoroughly examine the differential treatment response of vitamin $\mathrm{D}$ supplementation in different subsets of patients with knee OA. The results of this project have a high potential to provide important evidence to guide subgroup-specific treatment decisions in clinical practice to improve therapeutic effectiveness.

\section{Status of project}

Currently, literature search in the electronic databases has been completed.

\section{Author affiliations}

${ }^{1}$ Zhujiang Hospital, Southern Medical University, Guangzhou, China

${ }^{2}$ Centre for Big Data Research in Health, University of New South Wales, Sydney, New South Wales, Australia

${ }^{3}$ Institute of Bone and Joint Research, The University of Sydney, Sydney, New South Wales, Australia

${ }^{4}$ Menzies Institute for Medical Research, University of Tasmania, Hobart, Tasmania, Australia

${ }^{5}$ Centre of Evidence Based Dermatology, University of Nottingham, Nottingham, United Kingdom

${ }^{6}$ Tufts Medical Center, Boston, Massachusetts, USA

${ }^{7}$ Nuffield Department of Orthopaedics, Rheumatology and Musculoskeletal Sciences, University of Oxford, Oxford, United Kingdom

${ }^{8}$ MRC Lifecourse Epidemiology Unit, Southampton General Hospital, University of Shouthampton, Southampton, United Kingdom

${ }^{9}$ Department of Orthopaedic Surgery, King George's Medical University, Lucknow, India

${ }^{10}$ Department of General Practice, Erasmus MC University Medical Center, Rotterdam, The Netherlands

${ }^{11}$ Arthritis Research UK Pain Centre, University of Nottingham, Nottingham, United Kingdom

${ }^{12}$ Department of Epidemiology and Preventative Medicine, Monash University, Melbourne, Victoria, Australia

Twitter Monica SM Persson @MonicaPersson19

Acknowledgements We acknowledge the University of Sydney China Study Centre for providing short-term travel support to establish international collaboration for this project. 
Contributors All authors were involved in the study design and all will contribute to the interpretation of the results. $\mathrm{CD}, \mathrm{BA}$ and $\mathrm{XJ}$ concepted this study. WZ and MSMP provided methodological support for statistical analysis. XJ and BA will contact the potential data delivers and will coordinate the project. CD, FC, TM, NKA, RS and SS will deliver relevant data from their completed trials. SMAB-Z and MVM will facilitate legal agreement for data transfer and provide database management at the OA Trial Bank. XJ, BA and XW will perform the study selection, risk of bias assessment, data collection and analysis. All authors contributed to writing of this manuscript and approved the final submission.

Funding The authors have not declared a specific grant for this research from any funding agency in the public, commercial or not-for-profit sectors.

\section{Competing interests None declared.}

Patient and public involvement Patients and/or the public were not involved in the design, or conduct, or reporting, or dissemination plans of this research.

\section{Patient consent for publication Not required.}

Provenance and peer review Not commissioned; externally peer reviewed.

Open access This is an open access article distributed in accordance with the Creative Commons Attribution Non Commercial (CC BY-NC 4.0) license, which permits others to distribute, remix, adapt, build upon this work non-commercially, and license their derivative works on different terms, provided the original work is properly cited, appropriate credit is given, any changes made indicated, and the use is non-commercial. See: http://creativecommons.org/licenses/by-nc/4.0/.

ORCID iDs

Xingzhong Jin http://orcid.org/0000-0003-4293-8665

Monica SM Persson http://orcid.org/0000-0002-8532-3006

\section{REFERENCES}

1 Vos T, Allen C, Arora M, et al. Global, regional, and national incidence, prevalence, and years lived with disability for 310 diseases and injuries, 1990-2015: a systematic analysis for the global burden of disease study 2015. The Lancet 2016;388:1545-602.

2 Australian Bureau of Statistics. 2014-15 National health survey. Canberra: Australian Bureau of Statistics, 2017.

3 Ackerman IN, Pratt C, Gorelik A, et al. Projected burden of osteoarthritis and rheumatoid arthritis in Australia: a population-level analysis. Arthritis Care Res 2018;70:877-83.

4 Vos T, Flaxman AD, Naghavi M, et al. Years lived with disability (YLDs) for 1160 sequelae of 289 diseases and injuries 1990-2010: a systematic analysis for the global burden of disease study 2010 . The Lancet 2012;380:2163-96.

5 Kraus VB, Blanco FJ, Englund M, et al. Call for standardized definitions of osteoarthritis and risk stratification for clinical trials and clinical use. Osteoarthritis Cartilage 2015;23:1233-41.

6 Australian Orthopaedic Association National Joint Replacement Registry. Annual report 2012. Adelaide, Australia: AOA, 2012

7 Cao Y, Winzenberg T, Nguo K, et al. Association between serum levels of 25-hydroxyvitamin D and osteoarthritis: a systematic review. Rheumatology 2013;52:ket132:1323-34.

8 Garfinkel RJ, Dilisio MF, Agrawal DK. Vitamin D and its effects on articular cartilage and osteoarthritis. Orthop J Sports Med 2017;5:232596711771137.

9 Zhang FF, Driban JB, Lo GH, et al. Vitamin D deficiency is associated with progression of knee osteoarthritis. J Nutr 2014;144:2002-8.

10 Bellido M, Lugo L, Roman-Blas JA, et al. Improving subchondra bone integrity reduces progression of cartilage damage in experimental osteoarthritis preceded by osteoporosis. Osteoarthritis Cartilage 2011;19:1228-36.

11 Wang X, Cicutinni F, Jin X, et al. Effect of vitamin D on effusionsynovitis in knee osteoarthritis: a randomized controlled trial. Arthritis Rheumatol 2015;67:439-40.

12 Sharma A, Kudesia P, Shi Q, et al. Anxiety and depression in patients with osteoarthritis: impact and management challenges. Open Access Rheumatol Res Rev 2016;8:103-13.

13 Zheng S, Tu L, Cicuttini F, et al. Effect of vitamin D supplementation on depressive symptoms in patients with knee osteoarthritis. J Am Med Dir Assoc 2019;20:1634-40.

14 Arden NK, Cro S, Sheard S, et al. The effect of vitamin D supplementation on knee osteoarthritis, the video study: a randomised controlled trial. Osteoarthritis Cartilage 2016;24:1858-66.

15 McAlindon T, LaValley M, Schneider E, et al. Effect of vitamin $\mathrm{D}$ supplementation on progression of knee pain and cartilage volume loss in patients with symptomatic osteoarthritis. JAMA 2013;309:155-62.

16 Sanghi D, Mishra A, Sharma AC, et al. Does vitamin D improve osteoarthritis of the knee: a randomized controlled pilot trial. Clin Orthop Relat Res 2013;471:3556-62.

17 Jin X, Jones G, Cicuttini F, et al. Effect of vitamin D supplementation on tibial cartilage volume and knee pain among patients with symptomatic knee osteoarthritis: a randomized clinical trial. 2016: 315, 1005. http://doi.org/10.1001/jama.2016.1961

18 Hussain S, Singh A, Akhtar M, et al. Vitamin D supplementation for the management of knee osteoarthritis: a systematic review of randomized controlled trials. Rheumatol Int 2017;37:1489-98.

19 Diao N, Yang B, Yu F. Effect of vitamin D supplementation on knee osteoarthritis: a systematic review and meta-analysis of randomized clinical trials. Clin Biochem 2017;50:1312-6.

20 Felson DT. An update on the pathogenesis and epidemiology of osteoarthritis. Radiol Clin North Am 2004;42:1-9.

21 Wang X, Cicuttini F, Jin X, et al. Knee effusion-synovitis volume measurement and effects of vitamin $D$ supplementation in patients with knee osteoarthritis. Osteoarthritis Cartilage 2017;25:1304-12.

22 Groenwold RHH, Donders ART, van der Heijden GJMG, et al. Confounding of subgroup analyses in randomized data. Arch Intern Med 2009;169:1532-4.

23 Jørgensen L, Paludan-Müller AS, Laursen DRT, et al. Evaluation of the Cochrane tool for assessing risk of bias in randomized clinical trials: overview of published comments and analysis of user practice in Cochrane and non-Cochrane reviews. Syst Rev 2016;5:80.

24 Charlesworth J, Fitzpatrick J, Perera NKP, et al. Osteoarthritis- a systematic review of long-term safety implications for osteoarthritis of the knee. BMC Musculoskelet Disord 2019;20:151.

25 van Middelkoop M, Arden NK, Atchia I, et al. The oa trial bank: metaanalysis of individual patient data from knee and hip osteoarthritis trials show that patients with severe pain exhibit greater benefit from intra-articular glucocorticoids. Osteoarthritis Cartilage 2016;24:1143-52.

26 McConnell S, Kolopack P, Davis AM. The Western Ontario and McMaster universities osteoarthritis index (WOMAC): a review of its utility and measurement properties. Arthritis Rheum 2001;45:453-61.

27 Roos EM, Toksvig-Larsen S. Knee injury and Osteoarthritis Outcome Score (KOOS) - validation and comparison to the WOMAC in total knee replacement. Health Qual Life Outcomes 2003;1:17.

28 Bellamy N. WOMAC osteoarthritis index: user guide IV, 2000.

29 Pham T, van der Heijde D, Altman RD, et al. OMERACT-OARSI initiative: osteoarthritis research Society international set of Responder criteria for osteoarthritis clinical trials revisited. Osteoarthritis Cartilage 2004;12:389-99.

30 Janssen MF, Pickard AS, Golicki D, et al. Measurement properties of the EQ-5D-5L compared to the EQ-5D-3L across eight patient groups: a multi-country study. Qual Life Res 2013;22:1717-27.

31 Rat A-C, Coste J, Pouchot J, et al. OAKHQOL: a new instrument to measure quality of life in knee and hip osteoarthritis. J Clin Epidemiol 2005;58:47-55.

32 Kellgren JH, Lawrence JS. Radiological assessment of osteoarthrosis. Ann Rheum Dis 1957;16:494-502.

33 Altman RD, Gold GE. Atlas of individual radiographic features in osteoarthritis, revised. Osteoarthritis Cartilage 2007;15 Suppl A:A1-56.

34 Gudbergsen H, Lohmander LS, Jones G, et al. Correlations between radiographic assessments and $\mathrm{MRI}$ features of knee osteoarthritis--a cross-sectional study. Osteoarthritis Cartilage 2013;21:535-43.

35 Scragg R. Emerging evidence of thresholds for beneficial effects from vitamin D supplementation. Nutrients 2018;10:561.

36 Hunter DJ, Lo GH, Gale D, et al. The reliability of a new scoring system for knee osteoarthritis MRI and the validity of bone marrow lesion assessment: BLOKS (Boston Leeds osteoarthritis knee score). Ann Rheum Dis 2008;67:206-11.

37 Peterfy CG, Guermazi A, Zaim S, et al. Whole-Organ magnetic resonance imaging score (WORMS) of the knee in osteoarthritis. Osteoarthritis Cartilage 2004;12:177-90.

38 Kornaat PR, Ceulemans RYT, Kroon HM, et al. MRI assessment of knee osteoarthritis: Knee Osteoarthritis Scoring System (KOSS)-inter-observer and intra-observer reproducibility of a compartmentbased scoring system. Skeletal Radiol 2005;34:95-102.

39 Kroenke K, Spitzer RL, Williams JBW. The PHQ-9. J Gen Intern Med 2001;16:606-13.

40 Montorio I, Izal M. The geriatric depression scale: a review of its development and utility. Int Psychogeriatr 1996;8:103-12.

41 IntHout J, loannidis JPA, Borm GF. The Hartung-Knapp-SidikJonkman method for random effects meta-analysis is straightforward and considerably outperforms the standard DerSimonian-Laird method. BMC Med Res Methodol 2014;14:25. 
42 Higgins JPT, Thomas J, Chandler J, et al. Cochrane Handbook for systematic reviews of interventions version 6.0, 2019. Available: www.training.cochrane.org/handbook

43 Higgins JPT, Thompson SG, Deeks JJ, et al. Measuring inconsistency in meta-analyses. BMJ 2003;327:557-60.

44 Furuya-Kanamori L, Barendregt JJ, Doi SAR. A new improved graphical and quantitative method for detecting bias in metaanalysis. Int J Evid Based Healthc 2018;16:195-203.

45 van BS, Groothuis-Oudshoorn K. Mice: multivariate imputation by Chained equations in R. J Stat Softw 2011;45.

46 Burke DL, Ensor J, Riley RD. Meta-Analysis using individual participant data: one-stage and two-stage approaches, and why they may differ. Stat Med 2017;36:855-75.

47 Altman R, Asch E, Bloch D, et al. Development of criteria for the classification and reporting of osteoarthritis. classification of osteoarthritis of the knee. diagnostic and therapeutic criteria Committee of the American rheumatism association. Arthritis Rheum 1986;29:1039-49.

48 Gurevitch J, Koricheva J, Nakagawa S, et al. Meta-Analysis and the science of research synthesis. Nature 2018;555:175-82.

49 Cahan A, Cimino JJ. Improving precision medicine using individual patient data from trials. CMAJ 2017;189:E204-7.
50 Stewart LA, Tierney JF. To IPD or not to IPD? advantages and disadvantages of systematic reviews using individual patient data. Eval Health Prof 2002;25:76-97.

51 Reade MC, Delaney A, Bailey MJ, et al. Bench-to-bedside review: avoiding pitfalls in critical care meta-analysis--funnel plots, risk estimates, types of heterogeneity, baseline risk and the ecologic fallacy. Crit Care 2008;12:220.

52 Idrovo AJ. Three criteria for ecological fallacy. Environ Health Perspect 2011;119:a332.

53 Felson DT. Identifying different osteoarthritis phenotypes through epidemiology. Osteoarthritis Cartilage 2010;18:601-4.

54 Deveza LA, Melo L, Yamato TP, et al. Knee osteoarthritis phenotypes and their relevance for outcomes: a systematic review. Osteoarthritis Cartilage 2017;25:1926-41.

55 van Spil WE, Jansen NWD, Bijlsma JWJ, et al. Clusters within a wide spectrum of biochemical markers for osteoarthritis: data from check, a large cohort of individuals with very early symptomatic osteoarthritis. Osteoarthritis Cartilage 2012;20:745-54.

56 Riley RD, Lambert PC, Staessen JA, et al. Meta-Analysis of continuous outcomes combining individual patient data and aggregate data. Stat Med 2008;27:1870-93. 